\title{
Using New 3D CLEM Imaging Technique to Investigate the Effects of Substrate Mechanics on Cellular Uptake of Nanoparticle
}

\author{
Ya-Chen Chuang ${ }^{1,2}$, Wan-Yi Yen ${ }^{3}$, Linxi Zhang ${ }^{1,2}$, Wadie Bahou ${ }^{3}$, Marcia Simon ${ }^{4}$, Miriam Rafailovich ${ }^{1}$, \\ and Chung-Chueh Chang ${ }^{2 *}$ \\ 1. Department of Materials Science and Engineering, Stony Brook University, Stony Brook, NY 11794, \\ USA \\ 2. ThINC Facility, Advanced Energy Center, Stony Brook University, Stony Brook, NY 11794, USA \\ 3. Stem Cell Facility, Department of Medicine, Stony Brook University, Stony Brook, NY 11794, USA \\ 4. Department of Oral Biology and Pathology, Stony Brook University, Stony Brook, NY 11794, USA
}

In the past years, there have been many new developments in biological imaging, ranging from synchrotron X-ray crystallography for protein structure to 3D maps of entire human body [1]. Confocal microscopy, one of the most rapidly growing areas, is commonly used to construct the 3D images of biological samples where the area of interest is stained with fluorescent dyes conjugated to specific targeting antibodies. However, the physical resolution limit of light microscopy $(\sim 0.2 \mu \mathrm{m})$ does not permit visualization of features that are on the nanoscale. Transmission electron microscopy, TEM, is another popular technique where serial sectioning has permitted 3D reconstruction of biological elements. However, this technique is very difficult and requires extremely accurate positioning of the sections, which are each floated from the air/water interface. Lately, focused ion beam (FIB), has been introduced as a viable alternative, which can produce rapid and precise milling (sectioning) of the sample. In combination with scanning electron microscopy (SEM), it becomes a straightforward and powerful tool for 3D imaging of biological samples. Yet, despite its accuracy and high resolution, the EM techniques are not able to uniquely identify the proteins, which comprise the observed structures, or functional domains and receptors which determine the structure of the adhered cells. Hence immunohistochemical staining with fluorescently labeled antibodies has recently been used in combination with electron microsopy, otherwise known as, correlative light and electron microscopy (CLEM). It is a convenient technique which can be used to obtain comprehensive 3D images of tissues elucidating structure, composition, and cell lineage.

An elegant application of CLEM regards the influence of nanoparticles on tissue composition and development. Nanoparticles have been applied to drug delivery systems, targeted organ imaging, as well as cancer therapy [2]. Numerous evidences have shown that toxicological influence is not only on cell viability but on protein expression, extracellular matrix production, and cell mechanics. It has also been shown that substrate mechanics/ or topography can have influence on the cellular uptake of nanoparticles [3]. However, nanoparticle uptake as well as cell/substrate interactions can have very large variabilities in samples. Previous reports of these factors have been ensemble averages, making it very difficult to obtain the correlation between nanoparticle uptake in a specific cell and the consequent effect on a specific response. Furthermore, nanoparticles can only be imaged using electron microscopy, following sectioning and embedding, which requires removal of the cells from their substrates, impeding the ability to observe the response from cells to substrate mechanics. Hence, we developed a new approach, which is different from coventional CLEM, permits us to investigate the correlation between targeting protein expression of the cell, particle distributions, and particle uptake at the single cell level, where cells remain directly contact with substrate mechanics. This new approach can therefore be used to characterize multiple aspects of biological specimen on multiple length scales. 
In this study, human dermal fibroblasts were first cultured on different stiffness of polybutadiene (PB) spun-cast films, whose surface mechanics have previously shown continuous and differential of the change in modulus with film thickness and cells were able to adjust their moduli in a continuous manner in response to that of the substrate [4]. $\mathrm{TiO} 2$ nanoparticles were further added into cell cultures after cells sense and respond to underlying substrate stiffness. Cells on substrate were then fixed and stained with Alexa Fluor 488 Phalloidin and DAPI to examine the state of the actin filaments and the location of nuclei by using confocal microscopy. The same substrate was then followed by EM specimen staining procedure for FIB-SEM to collect data to reconstruct 3D imaging in which the identical cell was located by recognized features and relative coordinates from the edge of the substrate. The processed correlative 3D confocal and FIB-SEM images then allows to investigate the influence of surface mechanics on the cellular uptake of the nanoparticles as well as locating the marked actin filaments and nuclei at EM resolution.

In conclusion, we have demonstrated a promising new approach that permits accurate investigation of the influence of substrate mechanics on cell-particle interactions. Preliminary results, where this approach has also been extended to dental pulp stem cells will be presented. This approach is an even more critical tool for stem cell studies, since they are even more sensitive to environmental signals in their niche. Hence only a technique where the niche and cell positon within it, is preserved, is key to the success. All these advantages lead this tool to be very potential on many other applications where substrate effects are to be concerned.

\section{References:}

[1] K Narayan, S Subranmaniam, Nature Methods 12 (2015), p. 1021.

[2] A Dayem, et al, Biotechnol. J. 11 (2016), p. 1.

[3] C Huang, et al, Nano Lett. 13 (2013), p. 1611.

[4] C Chang, et al, Ann J Materials Sci Eng 1 (2014), p. 1.

[5] The authors acknowledge funding from the NSF-INSPIRE program and the ThINC facility at Advanced Energy Research and Technology Center.

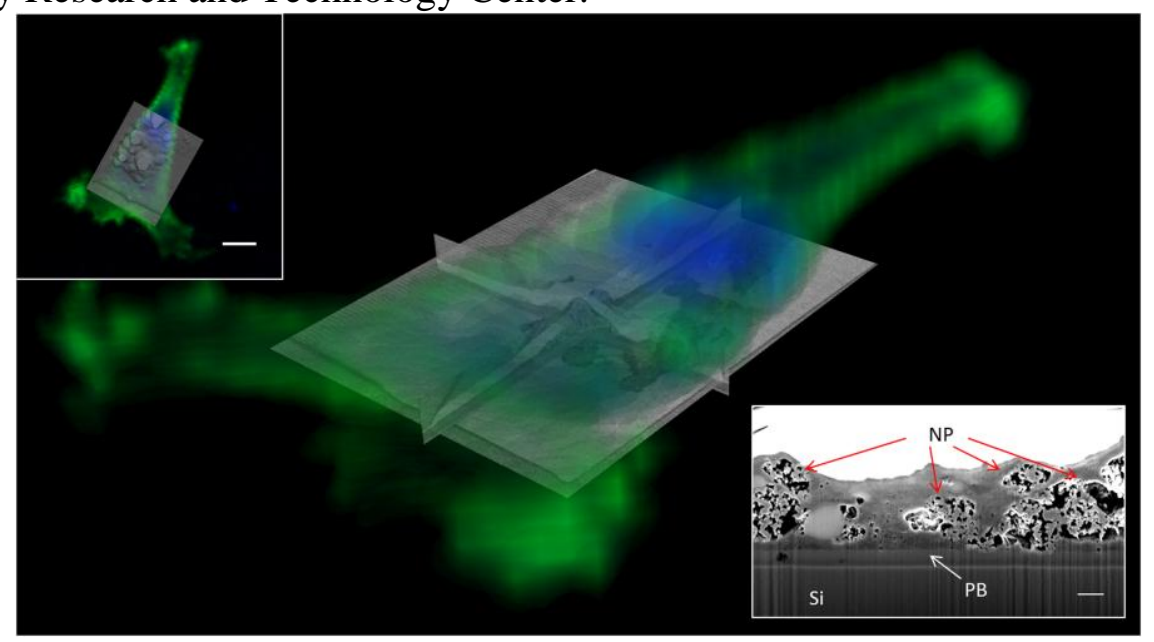

Figure 1. 3D CLEM shows the subcellular location of nanoparticles in single cell grown on spun-cast PB film on silicon wafer ( $\mathrm{Si}$ ). The top left inert shows the correlation of confocal and FIB-SEM images where scale bar is $15 \mu \mathrm{m}$. The bottom right inert reveals the cellular uptake of cluster of nanoparticles (NP) around the organelles where scale bar is $400 \mathrm{~nm}$. 